\title{
Modelling the dynamics of two political parties in the presence of switching
}

\author{
F. Nyabadza' ${ }^{1}$, Tobge Yawo Alassey ${ }^{2}$ and Gift Muchatibaya ${ }^{3^{*}}$
}

${ }^{*}$ Correspondence:

giftmuchatibaya@gmail.com

${ }^{3}$ Department

of Mathematics, University

of Zimbabwe, P. Box MP

167, Mount Pleasant, Harare, Zimbabwe

Full list of author information is available at the end of the article

\begin{abstract}
This paper generalizes the model proposed by Misra, by considering switching between political parties. In the model proposed, the movements of members from political party $B$ to political party $C$ and vice versa, are considered but the net movement is considered by assuming that $\theta_{1}-\theta_{2}=\theta$ (a constant), which implies that the movement of members is either from party $B$ to party $C$ or from party $C$ to party $B$. In this paper we remodel these movements through switching functions to capture how individuals switch between parties. The results provide a more comprehensive synopsis of the dynamics between two political parties.
\end{abstract}

Keywords: Political parties, Modelling, Switching function, Steady states, Simulations

\section{Background}

In ecology, the term switching was first coined by Murdoch in 1969, to describe a scenario where a predator predominantly eats the most common type of prey, see Murdoch (1969) and is often accompanied by a change in the habitat Khan (2000). Prey switching however happens when a predator's preference for a particular type of prey increases as the prey increases in abundance. Any display by a predator, of prey switching behaviour, can significantly affect the stability of the system, coexistence of prey species and evolutionary diversification. Switching can however promote coexistence between prey species Abrams and Matsuda (2003). A classical example is the case where prey switching causes low predation for rare prey, thus aiding prey refugia that often leads to coexistence Gentleman et al. (1990).

More often than not, political parties compete for membership. Members often switch between political parties as preferences change, often as a result of change of leadership, policies and perceived gains Fieldhouse et al. (2007), Petersen (1991), Schofield and Sened (2005), Romero et al. (2009). This paper is motivated by the work in Misra (2012). A closer loot at the work in Misra (2012) shows that there were simplifying assumptions that made the mathematical model tractable but overlooking some essential elements such as switching. The parameters $\theta_{1}$ and $\theta_{2}$ model movements between political parties $B$ and $C$. The net shifting of members $\theta=\theta_{1}-\theta_{2}$ is considered to be constant resulting in a unidirectional movement of members from $B$ to $C$ and vice versa. In this paper,

(c) 2016 The Author(s). This article is distributed under the terms of the Creative Commons Attribution 4.0 International License (http://creativecommons.org/licenses/by/4.0/), which permits unrestricted use, distribution, and reproduction in any medium, provided you give appropriate credit to the original author(s) and the source, provide a link to the Creative Commons license, and indicate if changes were made. 
we relook at this assumption by introducing switching functions whose parameters are endogenous to the system.

The paper is arranged as follows: in "The Misra model" section, we generalize the Misra model by including switching functions. The stabilities of the steady states are presented in "Stability of steady states" section and the paper is concluded in "Conclusion" section.

\section{The Misra model}

The model uses principles of mathematical epidemiology to model the dynamics of the two political parties. In general, most dynamical social phenomenon may be modelled by using these epidemiological type differential equations, see for instance Petersen (1991), Alvarez and Nagler (2000), Burden (2004), Huckfeldt and Kohfeld (1992), Belenky and King (2007). Following Misra (2012), the model is based on the following system of equations:

$$
\begin{aligned}
& \frac{d V}{d t}=\mu N-\beta_{1} V \frac{B}{N}-\beta_{2} V \frac{C}{N}-\mu V, \\
& \frac{d B}{d t}=\beta_{1} V \frac{B}{N}-\theta_{1} B \frac{C}{N}+\theta_{2} C \frac{B}{N}-\mu B, \\
& \frac{d C}{d t}=\beta_{2} V \frac{C}{N}+\theta_{1} B \frac{C}{N}-\theta_{2} C \frac{B}{N}-\mu C .
\end{aligned}
$$

Here, the total number of population $N(t)$ which was assumed constant, was divided into three classes, namely; voters class $V$, political party $B$ and political party $C$. The parameters and model assumptions are given in Misra (2012).

The non-dimensionalised model was obtained by setting

$$
v=\frac{V}{N}, \quad b=\frac{B}{N}, \quad \text { and } \quad c=\frac{C}{N},
$$

so that

$$
\begin{aligned}
& \frac{d v}{d t}=\mu-\beta_{1} v b-\beta_{2} v c-\mu v, \\
& \frac{d b}{d t}=\beta_{1} v b-\left(\theta_{1}-\theta_{2}\right) b c-\mu b, \\
& \frac{d c}{d t}=\beta_{2} v c+\left(\theta_{1}-\theta_{2}\right) b c-\mu c .
\end{aligned}
$$

The model was then reduced to a 2-dimensional system. One of the simplifying assumption made in the paper was setting $\theta=\theta_{1}-\theta_{2}>0$. This then resulted in a system in which the individuals moved from party $B$ to party $C$.

In this note we revisit the model in Misra (2012) and consider the following functions

$$
\theta_{1}(b)=\frac{\hat{\theta}_{1}\left(1-e^{-\alpha_{1} b}\right)}{1+m e^{-\alpha_{1} b}} \quad \text { and } \quad \theta_{2}(c)=\frac{\hat{\theta}_{2}\left(1-e^{-\alpha_{2} c}\right)}{1+m e^{-\alpha_{2} c}}
$$

where $\alpha_{1}, \alpha_{2}, m, \hat{\theta_{1}}$ and $\hat{\theta_{2}}$ are positive constants, to capture the aspects of switching. The parameters $m, \alpha_{1}$ and $\alpha_{2}$ must be chosen such that $\theta_{1}$ and $\theta_{2}$ approach 1 when $b, c$ 
approach 1 . The parameter $m$ affects the position of the switching point and $\alpha_{1}, \alpha_{2}$ affect both the switching point and the rate at which the switching occurs.

A graph of the function $\theta_{1}$ for different values of $\alpha_{1}$ with $\hat{\theta}_{1}=1$ and $m=25$ is depicted by Fig. 1 .

Figure 1 shows switching increases with increasing values of $\alpha_{1}$. It is important to note that the graph of $\theta_{2}$ follows a similar pattern. The gradient function of $\theta_{1}$ is:

$$
\frac{d \theta_{1}}{d b}=\frac{\hat{\theta}_{1} \alpha_{1}(1+m) e^{-\alpha_{1} b}}{\left(1+m e^{-\alpha_{1} b}\right)^{2}} .
$$

This function gives the change of $\theta_{1}$ with respect to $b$. This shows how the switching changes with respect to the state variable. Figure 2 shows the change in switching with respect to $b$ for the chosen set of parameter values in the caption. The peak increases with increasing $\alpha_{1}$. This means that people leave party $B$ for party $C$ faster and after a while decreases. This is consistent with either Type I or Type II response functions in ecology.

Setting $\theta(t, b, c)=\theta_{1}-\theta_{2}=\frac{\hat{\theta}_{1}\left(1-e^{-\alpha_{1} b}\right)}{1+m e^{-\alpha_{1} b}}-\frac{\hat{\theta}_{2}\left(1-e^{-\alpha_{2} c}\right)}{1+m e^{-\alpha_{2} c}}$, system (2) reduces to $\frac{d b}{d t}=\beta_{1}(1-b-c) b-\theta(t, b, c) b c-\mu b, \quad \frac{d c}{d t}=\beta_{2}(1-b-c) c+\theta(t, b, c) b c-\mu c$.

Here $\theta(t, b, c)$ can either be positive or negative, thus allowing individuals to switch between political parties. Just as in Misra (2012), system (3) has four equilibria, a party free equilibrium $E_{0}=(0,0)$, single party equilibria $E_{1}=\left(1-\frac{\mu}{\beta_{1}}, 0\right)$ and $E_{2}=\left(0,1-\frac{\mu}{\beta_{2}}\right)$, whose existence is subject to $\beta_{1}>\mu$ and $\beta_{2}>\mu$ respectively and the



Fig. 1 Graphs of $\theta_{1}$ against $b$ for different values of $\alpha_{1}$ 


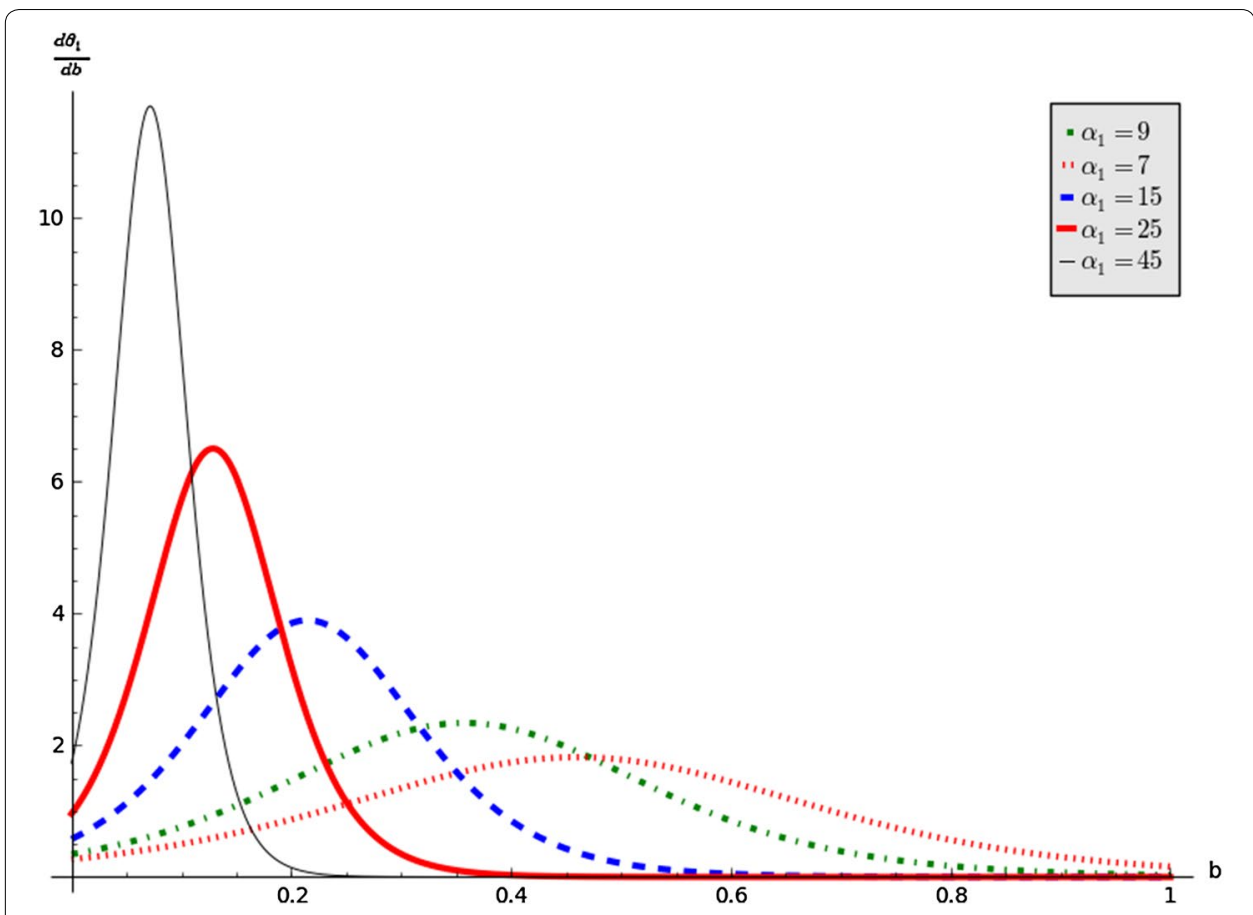

Fig. 2 Graphs of $\frac{d \theta_{1}}{d b}$ against $b$ by varying $\alpha_{1}$ and setting $\hat{\theta}_{1}=1$ and $m=25$

interior equilibrium. Unlike in Misra (2012), the interior equilibrium is only unique for the case $\theta_{1}(b)=\theta_{2}(c)$.

The graph of the switching function $\theta(t, b, c)$ against time is shown in Fig. 3. It is important note that the switching function is an increasing function that is initial negative until time $t s$ and then becomes positive there after. The interior equilibrium depends on the sign of switching function $\theta(t)$.

We consider three possible scenarios:

(1) The case $\theta(t, b, c)<0$. This is the case where members leave political party $C$ for $B$, for all $t<t$.

(2) The case $\theta(t, b, c)=0$.

(3) The case $\theta(t, b, c)>0$. This is the case considered in Misra (2012). This is the case where members leave political party $B$ for $C$, for all $t>t s$ where $t s$ is the time at which the switch occurs.

\section{Stability of steady states}

The stability of the boundary equilibria are presented in Misra (2012). If $\theta(t, b, c)=0$, we have

$$
\left\{\begin{array}{l}
\beta_{1}\left(1-b^{*}-c^{*}\right)-\mu=0, \\
\beta_{2}\left(1-b^{*}-c^{*}\right)-\mu=0,
\end{array} \Longrightarrow b^{*}+c^{*}=1-\frac{\mu}{\beta_{1}} \quad \text { and } \quad b^{*}+c^{*}=1-\frac{\mu}{\beta_{2}} .\right.
$$




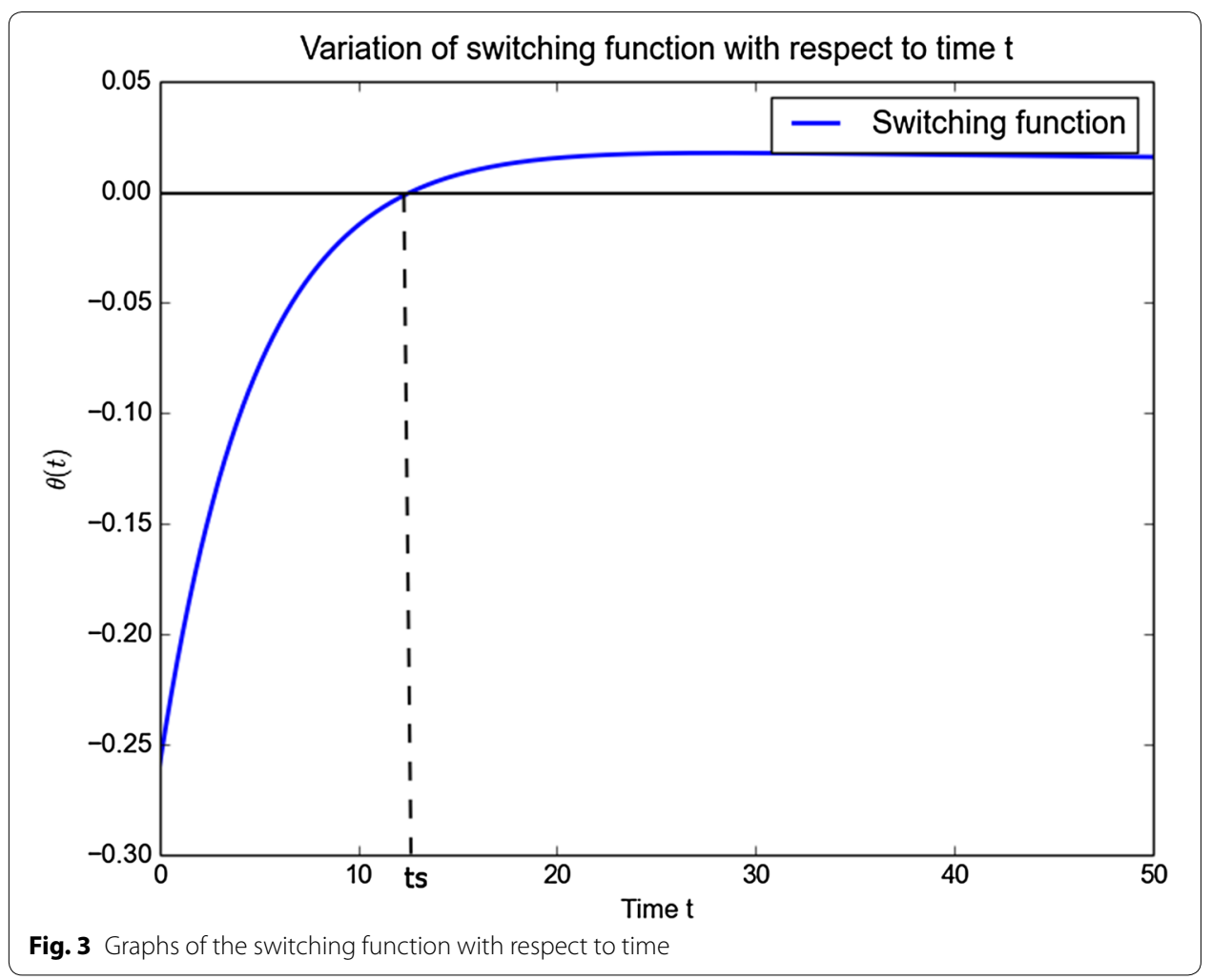

From this system (4), the values of $b^{*}$ and $c^{*}$ are obtained as follows:

- If $\beta_{1} \neq \beta_{2}$, then the interior equilibrium $E\left(b^{*}, c^{*}\right)$ does not exist.

- If $\beta_{1}=\beta_{2}$, then the interior equilibrium is stable and is a straight line satisfying the equation

$$
\left\{b^{*}, c^{*} \in[0,1] \mid b^{*}+c^{*}=1-\frac{\mu}{\beta_{1}}\right\} .
$$

The existence of the interior equilibrium is numerically given in Fig. 4.

Figure 4 shows the existence of the stable interior equilibrium which is a straight line that satisfies the equation

$$
\left\{b^{*}, c^{*} \in[0,1] \mid b^{*}+c^{*}=1-\frac{\mu}{\beta_{1}}\right\} \quad \text { when } \quad \beta_{1}=\beta_{2},
$$

whereas Fig. 5 shows the non-existence of the interior equilibrium when $\beta_{1} \neq \beta_{2}$.

We now consider the case when $\theta(t, b, c) \neq 0$. We begin by considering the plot in which political parties $B$ and $C$ coexist in a stable state. We come up with this result and both political parties start with an initial value of 0.2 at $t=0$.

A closer look at the Fig. 6 shows that there exists a time interval $t<t s$ where members of political party $C$ leave for political party $B$ when $\theta(t)<0$. After that members of political party $B$ leave for party $C$ before both become stable over the time. The corresponding interior equilibrium is shown in Fig. 7. 




Fig. $4 \beta_{1}=\beta_{2}=\frac{1}{24}$



Fig. $5 \beta_{1} \neq \beta_{2}, \beta_{1}=\frac{1}{24}, \beta_{2}=\frac{1}{36}$

An interesting aspect to consider is the switching by individuals that take place between political parties. To investigate such dynamics, we plot the variations of parties $B$ and $C$ as the parameters $\alpha_{1}, \hat{\theta}_{1}, \alpha_{2}$, and $\hat{\theta}_{2}$ are varied. The results are depicted in Figs. 8, 9, 10 and 11 . One can easily observe that increasing $\alpha_{1}$ and $\alpha_{2}$ leads to a decrease 

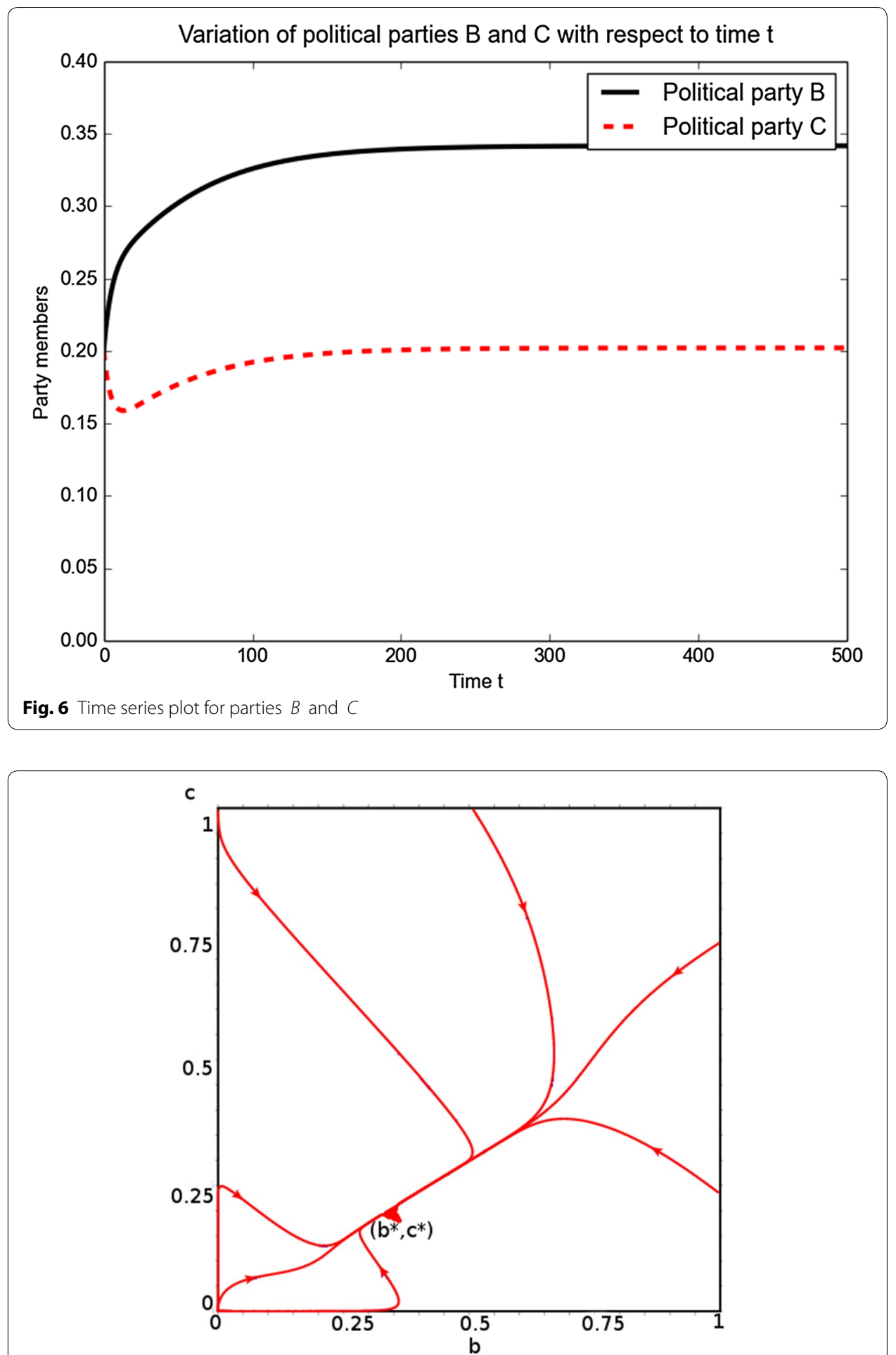

Fig. 7 Phase plot for the interior equilibrium $E\left(b^{*}, c^{*}\right)$

of membership in both political parties. So to maintain their numbers, political parties should focus on minimising any switching that can take place. A similar result is obtained for $\hat{\theta}_{1}$ and $\hat{\theta}_{2}$. Figures 8 and 9 show the evolution of political $B$ over the time by 

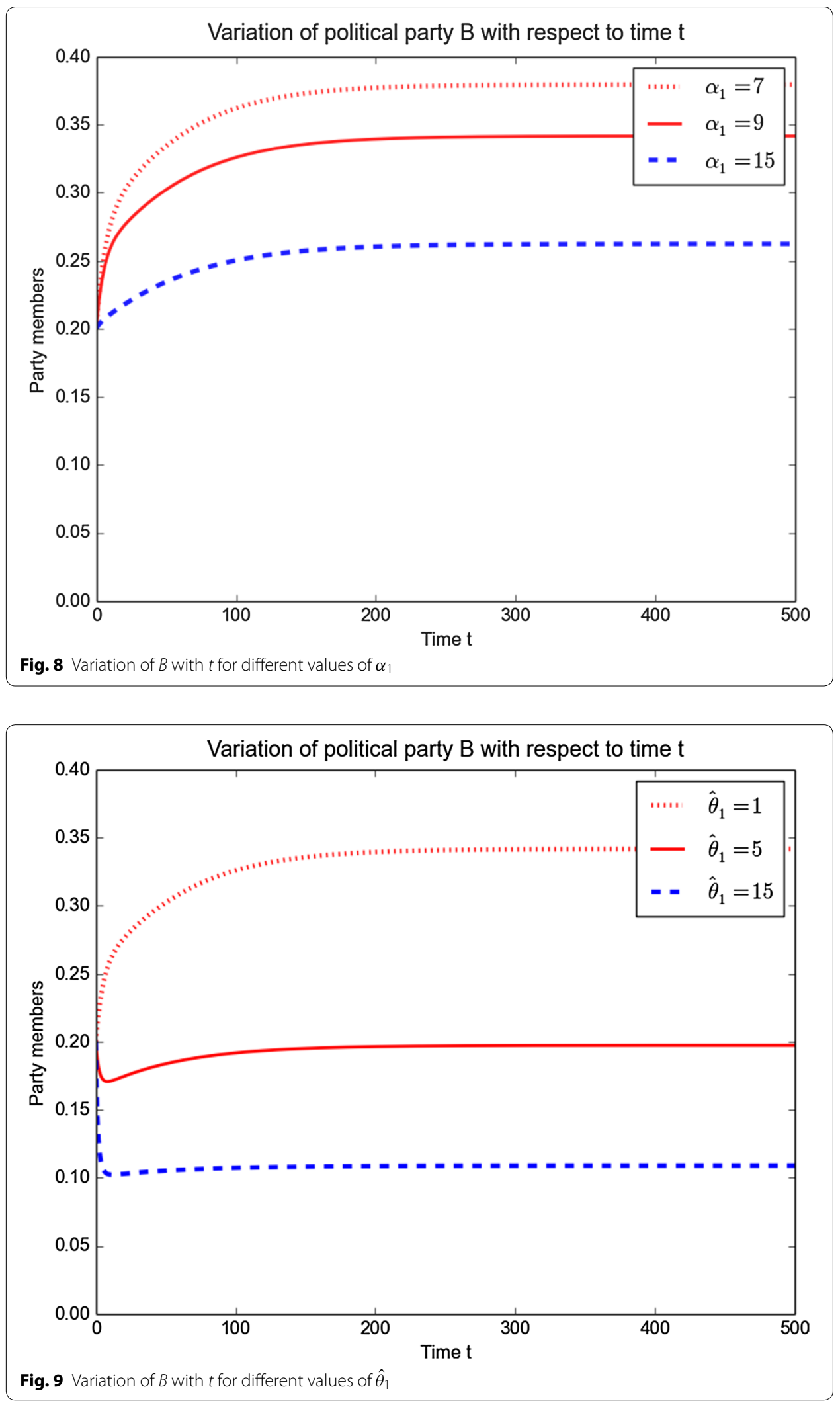


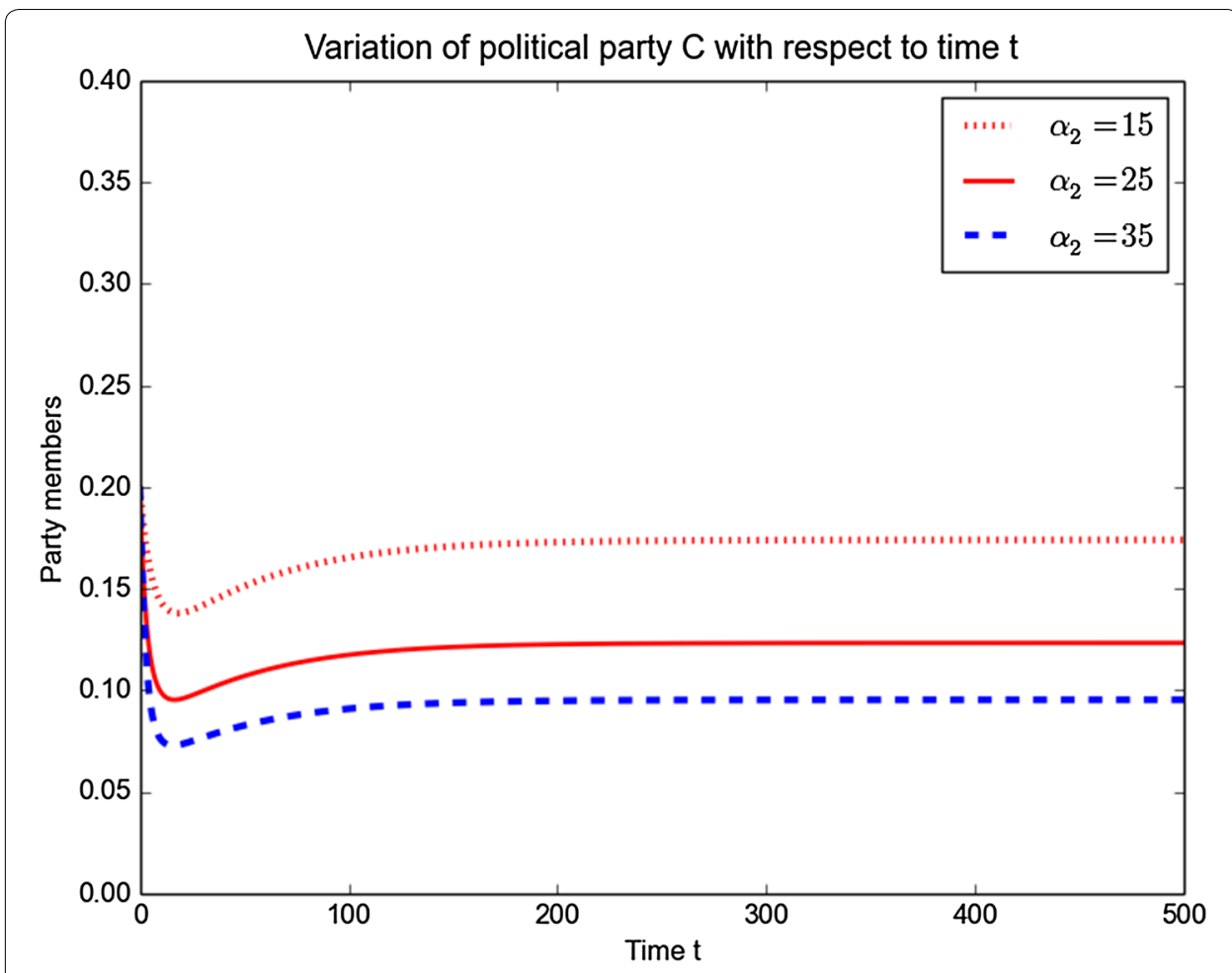

Fig. 10 Variation of $C$ with $t$ for different values of $\alpha_{2}$

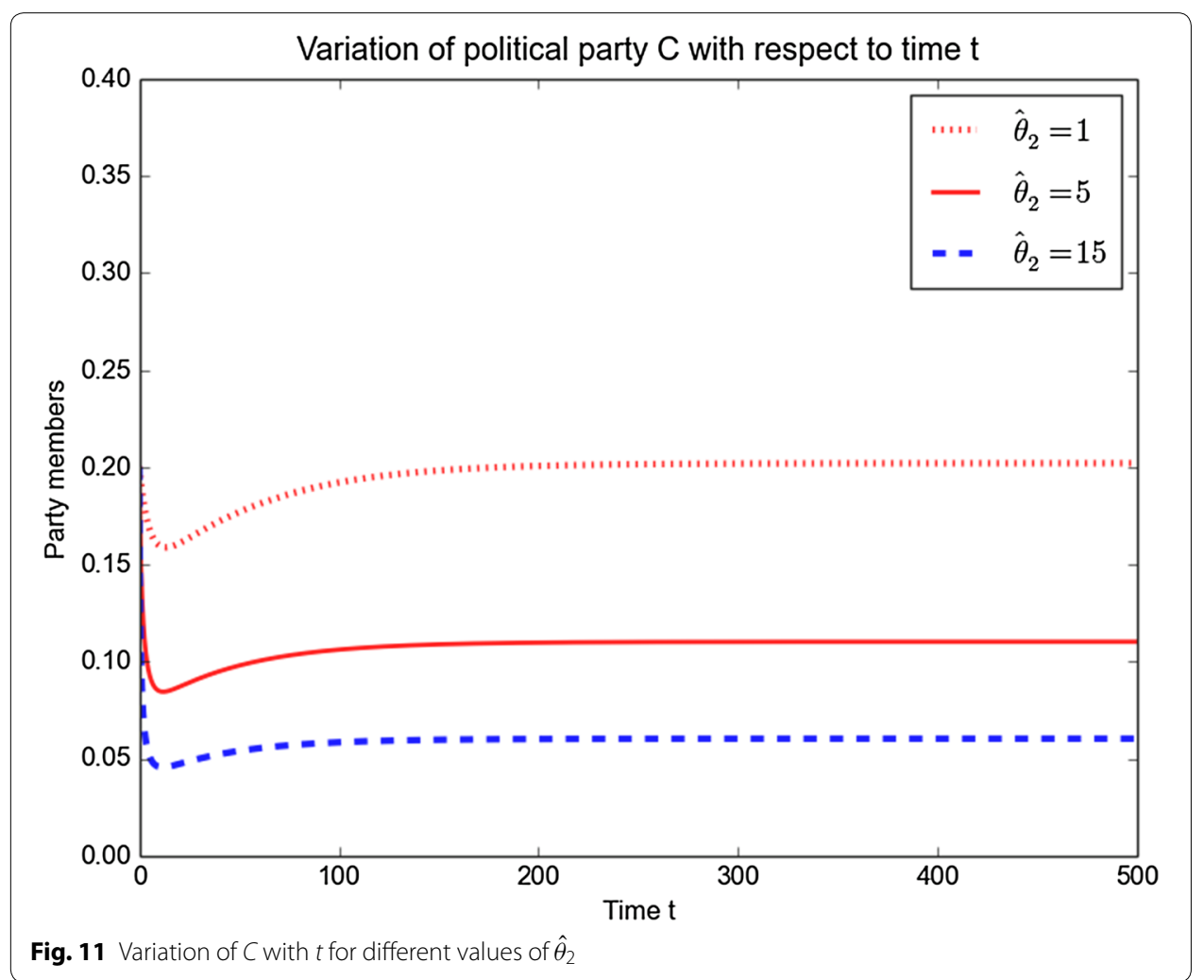


varying the parameter values $\alpha_{1}$ and $\hat{\theta}_{1}$ while keeping the other parameters constant. The figures show that when increasing the parameter values $\alpha_{1}$ and $\hat{\theta}_{1}$, the number of members of political $B$ decrease over the time.

Similar plots for political party $C$ for different values of $\alpha_{2}$ and $\hat{\theta}_{2}$ are shown in Figs. 10 and 11 . The figures show that when increasing the parameter values $\alpha_{2}$ and $\hat{\theta}_{2}$, leads to a decrease in the number of members of political party $C$ over the time.

\section{Conclusion}

In this paper, we remodelled switching between political parties in the model formulated in Misra (2012). This was achieved by removing the constraint that the difference between the net rates of movement between the two political parties be sign definite. We defined two switching functions that depend on the size of each political party and some parameters. These functions generalize the Misra paper in which the net movement was assumed to be unidirectional, or in favour of a given political party. In addition to some results obtained in Misra (2012), additional information regarding how the behaviour of the population size is dependent on the switching parameters is demonstrated.

The inclusion of switching functions in this paper improved the Misra (2012) model. There are further aspects that can be considered in future. Among these we mention the possibility of including individual preferences in choosing a political party. Another aspect will be the improvement of the model by considering a non constant population. An interesting aspect to consider is the age structured model, in view of the fact that political parties often target the youths for the future sustainability of the parties. There is however a trade off between mathematical tractability and realism. Finally, one can also look at how media companies influence the dynamics of political parties.

\section{Authors' contributions}

FN was instrumental in the conception and design of the model. TYA carried out the mathematical analysis and numerical simulations. GM did the mathematical analysis and all authors participated in writing and interpretation of numerical results of the manuscript. All authors read and approved the final manuscript.

\section{Author details}

${ }^{1}$ Department of Mathematical Science, University of Stellenbosch, Private Bag X1, Matieland 7602, South Africa. ${ }^{2}$ African Institute of Mathematical Sciences, Birwa, Ghana. ${ }^{3}$ Department of Mathematics, University of Zimbabwe, P. Box MP 167 , Mount Pleasant, Harare, Zimbabwe.

\section{Acknowledgements}

The first author acknowledges the support of Stellenbosch University in the production of the manuscript. The second author acknowledges the support of AIMS, Ghana. The third author acknowledges the support of the University of Zimbabwe.

Competing interests

The authors declare that they have no competing interests.

Received: 8 February 2016 Accepted: 1 June 2016

Published online: 08 July 2016

\section{References}

Abrams PA, Matsuda H (2003) Population dynamical consequences of reduced predator switching at low total prey densities. Deep Sea Res (II Top Stud Oceanogr) 50:2847-2875

Alvarez M, Nagler J (2000) A new approach for modelling strategic voting in multiparty elections. Br J Polit Sci 31(01):57-75

Belenky AS, King DC (2007) A mathematical model for estimating the potential margin of state undecided voters for a candidate in a us federal election. Math Comput Model 45(5):585-593

Burden BC (2004) Candidate positioning in us congressional elections. Br J Polit Sci 34(02):211-227 
Fieldhouse E, Shryane N, Pickles A (2007) Strategic voting and constituency context: modelling party preference and vote in multiparty elections. Polit Geogr 26(02):159-178

Gentleman W, Leisingb A, Frostc B, Stromd S, Murray J (1990) Functional responses for zooplankton feeding on multiple resources: a review of assumptions and biological dynamics. SIAM Rev 32(04):537-578

Huckfeldt R, Kohfeld CW (1992) Electoral stability and the decline of class in democratic politics. Math Comput Mode 16(08):223-239

Khan QJA (2000) Hopf bifurcation in multiparty political systems with time delay in switching. Appl Math Lett 13:43-52

Misra AK (2012) A simple mathematical model for the spread of two political parties. Nonlinear Anal Model Control 17(03):343-354

Murdoch WW (1969) Switching in generalist predators: experiments on prey specificity and stability of prey populations. Ecol. Monogr. 39:335-354

Petersen I (1991) Stability of equilibria in multi-party political systems. Math Soc Sci 21 (01):81-93

Romero DM, Kribs-Zaleta CM, Mubayi A, Orbe C (2009) An epidemiological approach to the spread of political third parties. Discret Contin Dyn Syst 15(03):707-738

Schofield N, Sened I (2005) Modeling the interaction of parties, activists and voters: why is the political center so empty? Eur J Polit Res 44(03):355-390

\section{Submit your manuscript to a SpringerOpen ${ }^{\circ}$ journal and benefit from:}

- Convenient online submission

\section{- Rigorous peer review}

- Immediate publication on acceptance

Open access: articles freely available online

- High visibility within the field

- Retaining the copyright to your article

Submit your next manuscript at $\gg$ springeropen.com 